\title{
Secular trends in the association of socio-economic position with self-reported dietary attributes and biomarkers in the US population: National Health and Nutrition Examination Survey (NHANES) 1971-1975 to NHANES 1999-2002†
}

\author{
Ashima K Kant ${ }^{1, *}$ and Barry I Graubard ${ }^{2}$ \\ 'Department of Family, Nutrition, and Exercise Sciences, Remsen Hall, Room 306E, Queens College of the City \\ University of New York, Flushing, NY 1 1367, USA: ${ }^{2}$ Division of Cancer Epidemiology and Genetics, Biostatistics \\ Branch, National Cancer Institute, National Institutes of Health, Bethesda, MD, USA
}

Submitted 27 October 2005: Accepted 3 April 2006

\begin{abstract}
Objective: Recent reports suggest persistence of health disparities related to socioeconomic position (SEP). To understand if diet may be a contributor to these trends, we examined secular trends in the association of diet and indicators of SEP from $1971-1975$ to $1999-2002$.

Design: We used data from the National Health and Nutrition Examination Surveys (NHANES) I (1971-1975), II (1976-1980), III (1988-1994) and 1999-2002 to examine the independent associations of poverty income ratio (PIR) and education with diet and biomarkers of diet and disease in $25-74$-year-olds $(n=36600)$. We used logistic and linear regression methods to adjust for multiple covariates and survey design to examine these associations.

Results: A large PIR differential in the likelihood of reporting a fruit or all five food groups and vitamin $\mathrm{C}$ intake, and an education differential in likelihood of obesity and carbohydrate intake, was noted in 1971-1975 but narrowed in 1999-2002 $(P<0.007)$. The positive association of education with intake of a fruit, vegetable or all five food groups, vitamins $\mathrm{A}$ and $\mathrm{C}$, calcium and potassium intake remained unchanged across surveys $(P<0.001)$. Similarly, the positive association of PIR with the amount of foods and intakes of energy and potassium remained unchanged over three decades $(P<0.001)$. The education and the PIR differential in energy density, and the PIR differential in the likelihood of obesity, persisted over the period of the four surveys $(P<0.001)$.

Conclusions: Persistence of unfavourable dietary and biomarker profiles in Americans with low income and education suggests continued need for improvement in the quality of diets of these high-risk groups.
\end{abstract}

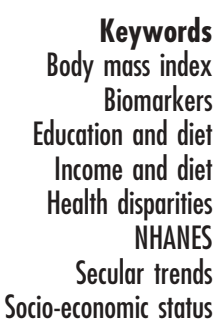

Measures of socio-economic position (SEP) such as education and family income have generally been shown to relate inversely with mortality and morbidity ${ }^{1-4}$. Recent reports suggest that the previously reported socioeconomic differential in all-cause, cardiovascular and cancer mortality and healthy life expectancy in the USA may be increasing over time ${ }^{5-9}$. Socio-economic disparities in health may be attributable to income, access to quality health care, environmental exposures, early life environment, lifestyle, and health risk behaviours including diet ${ }^{10-12}$.

Dietary factors contribute substantially to the burden of preventable illnesses and premature deaths in the USA.

†Preliminary results were presented at Experimental Biology 2005 held in San Diego, CA, USA on 2-6 April 2005.
Diet is a health risk behaviour which has been shown to relate to three of the leading causes of death in the USA (coronary heart disease, certain cancers and stroke) ${ }^{1}$. Diet also plays a major role in the development and management of morbidities such as obesity, diabetes, hypertension and osteoporosis ${ }^{1,13}$. Although it is generally believed that in affluent societies higher SEP may be associated with healthier dietary profiles, surprisingly little has been published on changes in the association of SEP and attributes of diet quality over time in the US population. To understand whether diet may be one of the explanatory variables for persistence of SEP disparity in health, we examined secular trends in the independent associations of two measures of SEP - education and income - with self-reported dietary attributes and biomarkers in the US population. 


\section{Materials and methods}

We used data from the National Health and Nutrition Examination Surveys (NHANES) I (1971-1975), II (19761980), III (1988-1994) and 1999-2002, conducted by the National Center for Health Statistics (NCHS) of the Centers for Disease Control and Prevention ${ }^{14}$. The NHANES I, II and III were multi-year surveys; beginning in 1999, the NHANES became a continuous annual survey and these data are being released for two years at a time. The sample design for each survey is a stratified, multistage, national probability sample of the USA. NHANES I and II sampled subjects aged 1 to 74 years; NHANES III sampled ages 2 months and older; and NHANES 1999-2002 included all ages. The survey procedures consisted of a household interview of the sample person conducted by a trained interviewer and a health examination in the mobile examination centre (MEC). Weight and height measurements, interview to collect a 24-hour dietary recall, and blood and urine specimens were obtained in the MEC. Survey response rates for examined (MEC) individuals for NHANES I, II, III and 1999-2000 were 74, 73, 78 and 76\%, respectively $^{14}$.

\section{Information on measures of SEP}

\section{Education}

Each NHANES collected information on the highest grade of education completed by the survey respondent. Ease of measurement, applicability to people not in the labour force, stability over adult lifespan and the fact that health status (or diet) does not influence level of education make it a powerful and widely used indicator of SEP in US public health research ${ }^{15,16}$. Due to unavailability of more detailed education information in the NHANES 1999-2002 public release dataset, in this study we operationalised the level of education as $<12,12$ and $>12$ years.

\section{Poverty income ratio}

For each NHANES, the NCHS has computed a poverty income ratio (PIR) based on the survey-year-specific poverty threshold from the US Census Bureau ${ }^{14}$. The PIR is a ratio of total family income to the poverty threshold for a family of given characteristics specific to each survey. A PIR of $<1$ suggests that income is below the poverty level. Although not without limitations, the PIR is a normative construct as it assesses income in relation to need adjusting for inflation. Various authors have argued that examining PIR as just below or above poverty provides little information about the relationship of income gradient with health ${ }^{15,16}$. Therefore, we operationalised the PIR variable as $<1.0,1.0-1.99,2.0-2.99,3.0-3.99$ and $\geq 4.0$.

\section{Dietary metbods}

All the NHANES collected dietary information using a 24hour dietary recall administered by a trained dietary interviewer in the $\mathrm{MEC}^{14}$. Beginning with NHANES III, trained dietary interviewers administered the 24-hour recall using an automated, interactive format with built-in probes to improve the quality of the recalls obtained. The dietary recalls collected for the NHANES 1999-2000 and 2001 survey years used a computer-assisted dietary interview which included a four-step multiple-pass approach $^{14}$. In NHANES 2002, the dietary data were collected using a multiple-pass approach with dietary recall methods that are part of the integrated US Department of Agriculture and NHANES 'What We Eat in America' protocol ${ }^{14}$.

\section{Dietary outcome variables}

To obtain a comprehensive picture of secular changes in diet and SEP associations, we examined two types of dietary attribute in this study. The first group may be considered to relate with reported quantity of food which in turn may relate with energy intake and body weight, and included total amount of reported foods and beverages, energy content and energy density of the reported diet. These variables are especially pertinent given recent reports suggesting that the portion sizes consumed by Americans have increased over the period of surveys examined in this study ${ }^{17}$. The energy density of diets is receiving considerable attention as a correlate of energy intake and body weight ${ }^{18-22}$, and is also believed to have changed over the past three decades. There is no consensus on how energy density should be defined ${ }^{19-21}$. The association of different energy density measures with nutrient profiles and body weight varies depending on how this variable is defined ${ }^{19-21}$. For example, the association of energy and nutrient intake with energy density variables that included beverages was not as strong as with energy density variables derived from solid foods ${ }^{21}$, possibly due to a different physiological mechanism of regulation of beverage intake as suggested by Rolls et al. ${ }^{22}$. Therefore, in the present study, we assessed dietary energy density (kcal per $\mathrm{g}$ ) of all foods and nutritive beverages (i.e. milk and 100\% juices, but excluding all alcoholic and non-alcoholic energy-yielding or non-energy-yielding beverages - e.g. coffee, tea, sodas, juice drinks).

The second group of dietary variables included foods/nutrients potentially related to health. Many nutrients with known associations with health - vitamin E, folate, dietary fibre and carotenoids - were not available for all surveys; therefore the nutrients examined were limited to those available in all surveys, and included intakes of fat and saturated fat, carbohydrate, and the micronutrients vitamins $\mathrm{C}$ and $\mathrm{A}$, calcium and potassium. Whether respondents mentioned any food from the fruit or vegetable group (as defined below) and a summary measure of overall diet quality - dietary diversity score $(D D S)^{23}$ - were also examined. The DDS, a relatively simple measure of diet quality, was shown to relate with mortality in the NHANES I epidemiologic follow-up study 
cohort $^{24}$ and with biomarkers in NHANES III ${ }^{25}$. The DDS considers whether or not a food from each of the five major food groups (fruit, vegetable, dairy, meat/alternative, and grain) was mentioned in the recall ${ }^{23}$. To compute the DDS, foods reported in each survey were grouped into one or more of the major food groups using methods described previously ${ }^{23}$. The fruit group included all fresh, frozen or canned fruits and 100\% juices, but excluded fruit drinks and fruit desserts such as fruit pies. The vegetable group included all raw, canned and frozen vegetables and juices. The grain group included all cereals, breads, pasta, rice, etc., but excluded pastries, cookies, cakes and pies. The meat group included eggs, meat, poultry, fish and alternatives such as beans, nuts and seeds. The dairy group included milk, yoghurt and cheese but excluded ice cream and other dairy desserts. Foods in mixed dishes were grouped into all the constituent food groups.

As an estimate of possible low energy reporting, a ratio of reported energy intake (EI) to basal energy expenditure (BEE) was also computed. The BEE was estimated using age-, sex- and weight-specific equations developed by the Dietary Reference Intakes committee ${ }^{26}$. We used an EI/BEE ratio of $<1.2$ to suggest low energy reporting in this study.

\section{Biomarkers}

We also examined trends in the association of SEP with three biomarkers: body mass index (BMI), serum total cholesterol (TC) and serum high-density lipoprotein cholesterol (HDL-C). Serum HDL-C was not available for NHANES I; these analyses included data from the latter three surveys.

\section{Analytic sample}

For each of the four surveys, all non-pregnant, nonlactating respondents aged 25-74 years, with a 24-hour dietary recall (considered reliable by the NCHS) and with information on PIR and education were included in the analytic sample. The lower age cut-off of 25 years was chosen because post-school education is usually completed by this age; the upper age cut-off of 74 years was necessary because NHANES I and II did not include respondents older than 74 years. The total sample size for the four surveys was 36600 (NHANES I = 10 065; NHANES $\mathrm{II}=9659 ; \quad$ NHANES $\quad$ III $=11002 ; \quad$ NHANES $\quad 1999-$ $2002=5874)$.

\section{Analytical methods}

We used linear or logistic multiple regression models to assess the independent association of education and PIR with dietary and biomarker outcomes. Because of differences in the distribution of a number of factors that may be associated with reporting of food intake among surveys, the regression models included gender, age, age ${ }^{2}$, race (white, black, other), PIR $(<1.0,1.0-1.99$, 2.0-2.99, $3.0-3.99$, $\geq 4.0)$, years of education $(<12,12,>12)$ and survey (NHANES I, NHANES II, NHANES III, NHANES
1999-2002) as independent variables with each dietary attribute or biomarker as a continuous or binary outcome. Regression models for predicting changes in the SEPbiomarker associations also included smoking status, alcohol use status and leisure-time physical activity as covariates. The race/ethnicity categories available in NHANES 1999-2002 differ markedly from race categories in earlier surveys. NHANES I and II provide only white, black and other categories. NHANES III provides both race- and ethnicity-specific categories. However, NHANES 1999-2002 provides only ethnicity-specific categories (non-Hispanic whites, non-Hispanic black, Mexican American, Other Hispanic and All other). Therefore, we categorised race as white, black and other as available in NHANES I, II and III. For NHANES 1999-2002, we grouped Mexican-Americans and other Hispanics with non-Hispanic whites. These methods are similar to those used by others ${ }^{27}$. To assess whether the association of education or PIR with dietary attributes differed among the four surveys, all models included two interaction terms: PIR by survey and education by survey. If the interaction term(s) was not significant, we examined the main effects of education and PIR after exclusion of the interaction term(s), across all surveys combined. A backwards stepwise regression with an exclusion criterion of $P>0.01$ for the interaction was used to determine which interactions remained in the model. The mean estimates presented in the tables and figures are predictive margins obtained from fully adjusted regression models ${ }^{28}$. In tests for trend and interaction, survey, education and PIR were modelled as continuous variables.

Because we combined the data from four surveys for these analyses, our approach was to treat the data from the four different surveys as independent samples from different populations. Therefore, we weighted the data in our analyses using the NCHS-assigned survey-specific sample weights so as to produce estimates that represented each population ${ }^{29}$. All statistical analyses were adjusted for the sample weights and complex sample design of the four national surveys by using SAS callable SUDAAN, version $9.0^{30}$. All $P$-values were two-sided.

Given the multiple tests of association done in this study and the large sample size, we chose a conservative criterion of $P<0.01$ for finding statistical significance. Although we present all results, the discussion is limited to variables where the level of significance was $P<0.01$.

\section{Results}

The percentage of those with $<12$ years of education decreased while the percentage with $>12$ years increased during this period (Table 1). The percentage of the population below a PIR of 1 and above a PIR of 4 increased from 1971-1975 to 1999-2002 (Table 1). Across all surveys combined, a higher proportion of those in lower PIR categories were female, non-white, aged 25-39 
Table 1 Characteristics (percentage \pm SE, weighted) of the surveyed populations: NHANES I, NHANES II, NHANES III and NHANES 1999-2002

\begin{tabular}{|c|c|c|c|c|c|}
\hline & All surveys & $\begin{array}{c}\text { NHANES } \\
\text { I (1971-1975) }\end{array}$ & $\begin{array}{c}\text { NHANES } \\
\text { II }(1976-1980)\end{array}$ & $\begin{array}{c}\text { NHANES } \\
\text { III (1988-1994) }\end{array}$ & $\begin{array}{c}\text { NHANES } \\
1999-2002\end{array}$ \\
\hline$n$ & 36600 & 10065 & 9659 & 11002 & 5874 \\
\hline Females & $51.3 \pm 0.3$ & $53.1 \pm 0.6$ & $51.9 \pm 0.5$ & $50.7 \pm 0.5$ & $50.2 \pm 0.6$ \\
\hline \multicolumn{6}{|l|}{ Race } \\
\hline White & $87.0 \pm 0.6$ & $89.4 \pm 0.7$ & $88.0 \pm 1.5$ & $85.5 \pm 0.8$ & $85.9 \pm 1.2$ \\
\hline Black & $10.1 \pm 0.5$ & $9.7 \pm 0.7$ & $9.7 \pm 1.2$ & $10.8 \pm 0.6$ & $10.2 \pm 1.1$ \\
\hline Others & $2.9 \pm 0.3$ & $0.9 \pm 0.2$ & $2.2 \pm 0.9$ & $3.7 \pm 0.4$ & $3.9 \pm 0.5$ \\
\hline \multicolumn{6}{|l|}{ Age group } \\
\hline $25-39$ years & $38.5 \pm 0.5$ & $36.5 \pm 0.8$ & $39.7 \pm 0.9$ & $42.7 \pm 1.0$ & $34.9 \pm 1.2$ \\
\hline $40-59$ years & $42.3 \pm 0.4$ & $43.5 \pm 0.9$ & $39.3 \pm 0.6$ & $38.2 \pm 0.7$ & $47.5 \pm 1.0$ \\
\hline $60-74$ years & $19.2 \pm 0.4$ & $19.8 \pm 0.7$ & $21.0 \pm 0.6$ & $19.1 \pm 0.9$ & $17.5 \pm 0.7$ \\
\hline \multicolumn{6}{|c|}{ Poverty income ratio } \\
\hline$<1.0$ & $10.7 \pm 0.3$ & $9.9 \pm 0.7$ & $9.9 \pm 0.5$ & $10.7 \pm 0.7$ & $11.7 \pm 0.7$ \\
\hline $1.0-1.99$ & $20.6 \pm 0.5$ & $24.3 \pm 0.9$ & $23.4 \pm 0.6$ & $18.8 \pm 0.7$ & $18.4 \pm 1.2$ \\
\hline $2.0-2.99$ & $20.8 \pm 0.4$ & $22.7 \pm 0.8$ & $24.8 \pm 0.7$ & $21.7 \pm 0.8$ & $15.7 \pm 1.8$ \\
\hline $3.0-3.99$ & $18.0 \pm 0.4$ & $18.4 \pm 24.7$ & $21.7 \pm 0.6$ & $18.9 \pm 1.0$ & $14.1 \pm 0.7$ \\
\hline$\geq 4.0$ & $29.7 \pm 0.7$ & $24.7 \pm 1.0$ & $20.1 \pm 0.8$ & $29.8 \pm 1.4$ & $40.0 \pm 1.9$ \\
\hline \multicolumn{6}{|c|}{ Years of education } \\
\hline$<12$ & $26.8 \pm 0.5$ & $38.2 \pm 1.1$ & $32.5 \pm 1.0$ & $22.1 \pm 1.1$ & $19.2 \pm 0.9$ \\
\hline 12 & $32.9 \pm 0.5$ & $36.6 \pm 0.8$ & $36.5 \pm 1.0$ & $34.9 \pm 0.8$ & $25.6 \pm 1.1$ \\
\hline$>12$ & $40.3 \pm 0.7$ & $25.2 \pm 1.1$ & $31.0 \pm 1.2$ & $43.0 \pm 1.2$ & $55.1 \pm 1.6$ \\
\hline
\end{tabular}

SE - standard error; NHANES - National Health and Nutrition Examination Survey.

years and with $<12$ years of education. Respondents with $<12$ years of education were similarly more likely to be non-white and in low PIR categories, but were more likely to be aged 60-74 years (data not shown; available from the authors on request).

Secular trends from 1971-1975 to 1999-2002 in the various outcomes examined are presented in Table 2. The percentage of the population with $\mathrm{BMI}>30$ $\mathrm{kg} \mathrm{m}^{-2}$, the covariate-adjusted means of serum HDL-C concentration, reported amount of all foods and beverages, energy density, and intakes of energy and carbohydrate increased across surveys. However, the adjusted means of serum TC concentration, total fat, saturated fat, and the percentage reporting a DDS of 5 or a fruit in the recall declined from 1971-1975 to 1999-2002.

Table 2 Adjusted mean $\pm \mathrm{SE}^{*}$ and prevalence of biomarkers and dietary attributes in the US population: NHANES I, NHANES II, NHANES III and NHANES 1999-2002

\begin{tabular}{|c|c|c|c|c|c|}
\hline & NHANES I & NHANES II & NHANES III & NHANES 1999-2002 & $P$ (trend) \\
\hline $\mathrm{BMI} \geq 30 \mathrm{~kg} \mathrm{~m}^{-2}(\%) \dagger$ & $15 \pm 0.6$ & $15 \pm 0.4$ & $24 \pm 0.9$ & $33 \pm 1$ & $<0.001$ \\
\hline Serum TC $\left(\mathrm{mg} \mathrm{dl}^{-1}\right) \dagger$ & $221 \pm 1.0$ & $219 \pm 1.1$ & $208 \pm 0.8$ & $204 \pm 1.1$ & $<0.001$ \\
\hline Serum HDL-C (mg dl $\left.{ }^{-1}\right) \dagger \ddagger$ & NA & $48.7 \pm 0.4$ & $50.7 \pm 0.3$ & $51.1 \pm 0.3$ & $<0.001$ \\
\hline Amount of all foods and beverages $(\mathrm{g})$ & $2275 \pm 23$ & $2317 \pm 19$ & $2575 \pm 23$ & $2617 \pm 25$ & $<0.001$ \\
\hline Energy (kcal) & $1937 \pm 18$ & $1927 \pm 16$ & $2188 \pm 17$ & $2228 \pm 14$ & $<0.001$ \\
\hline Energy density $\left(\mathrm{kcal} \mathrm{g}^{-1}\right) \S$ & $1.58 \pm 0.01$ & $1.60 \pm 0.01$ & $1.67 \pm 0.01$ & $1.71 \pm 0.02$ & $<0.001$ \\
\hline Carbohydrate $(\mathrm{g}) \boldsymbol{q}$ & $223 \pm 1$ & $225 \pm 1$ & $253 \pm 2$ & $260 \pm 1$ & $<0.001$ \\
\hline Total fat $(\mathrm{g})$ ฯ & $87 \pm 0.6$ & $87 \pm 0.6$ & $80 \pm 0.8$ & $78 \pm 0.6$ & $<0.001$ \\
\hline Saturated fat $(\mathrm{g})$ & $31 \pm 0.3$ & $31 \pm 0.2$ & $27 \pm 0.3$ & $25 \pm 0.2$ & $<0.001$ \\
\hline Vitamin C (mg) & $93 \pm 2$ & $106 \pm 2$ & $101 \pm 2$ & $88 \pm 3$ & 0.04 \\
\hline Vitamin A (IU)ף & $5559 \pm 141$ & $5913 \pm 100$ & $6721 \pm 148$ & $5291 \pm 159$ & 0.66 \\
\hline Calcium (mg) & $791 \pm 9$ & $780 \pm 10$ & $792 \pm 10$ & $799 \pm 8$ & 0.17 \\
\hline Potassium (mg) & $2496 \pm 19$ & $2642 \pm 18$ & $2816 \pm 17$ & $2656 \pm 23$ & $<0.001$ \\
\hline Mentioned a vegetable (\%) & $91 \pm 0.4$ & $90 \pm 0.4$ & $92 \pm 0.4$ & $92 \pm 0.5$ & 0.17 \\
\hline Mentioned a fruit (\%) & $60 \pm 0.8$ & $59 \pm 0.9$ & $52 \pm 0.8$ & $51 \pm 1.0$ & $<0.001$ \\
\hline $\mathrm{DDS}=5(\%) \|$ & $46 \pm 0.8$ & $43 \pm 0.8$ & $37 \pm 0.8$ & $37 \pm 1.0$ & $<0.001$ \\
\hline
\end{tabular}

SE - standard error; NHANES - National Health and Nutrition Examination Survey; BMI - body mass index; TC, total cholesterol; HDL-C - high-density lipoprotein cholesterol; DDS - dietary diversity score.

${ }^{*}$ From regression models that included gender, age, age ${ }^{2}$, race/ethnicity (white, black, other), years of education $(<12,12,>12)$, poverty income ratio $(<1.0,1.0-2.99,2.0-2.99,3.0-3.99, \geq 4.0)$ and survey (NHANES I, II, III, 1999-2002) $(n=36600)$.

† Models included BMI (for serum TC and HDL-C), smoking status (never, former, current), alcohol user (yes, no) and any leisure-time physical activity (yes, no) in addition to above variables. Included respondents with complete covariate information ( $n=34993$ for BMl; 33809 for serum TC; 23685 for serum HDL-C).

¥ HDL-C analyses were based on data from NHANES II, III and 1999-2002 (HDL-C data were not available (NA) for NHANES I).

$\S$ Energy density $=\mathrm{kcal} \mathrm{g}^{-1}$ of all reported foods and nutritive beverages.

I Models included energy intake (kcal).

II DDS based on consideration of mention of all five food groups (dairy, fruit, vegetable, grain, meat or alternative) in the recall. 


\section{Interactions of PIR and education with survey}

The interaction of education with survey was significant for carbohydrate intake $(P<0.001)$ and likelihood of obesity $(P=0.007)$ (Fig. 1$)$, suggesting a change in the association of these variables with education across surveys. Both of these variables were inversely associated with education in earlier surveys, but not
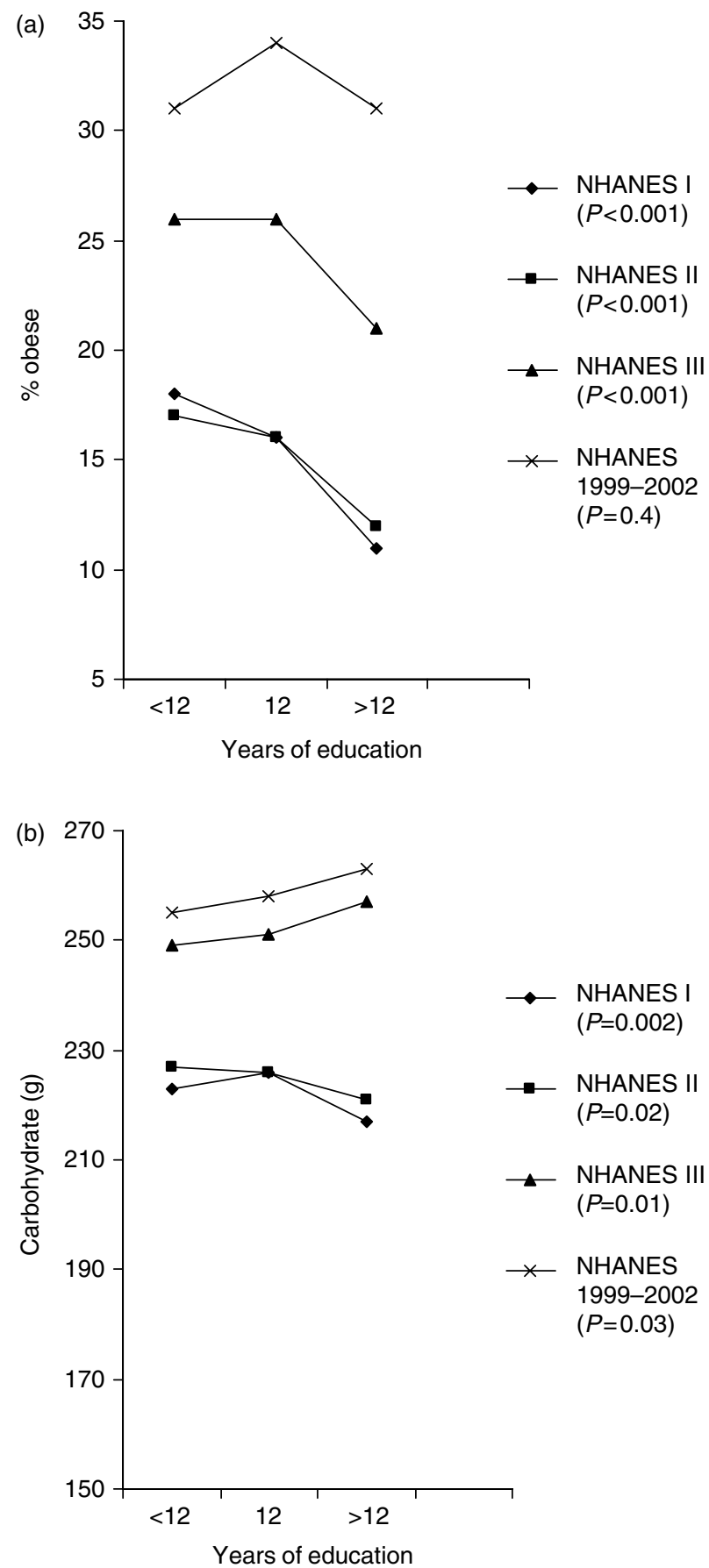

Fig. 1 Changes in the association of years of education with (a) prevalence of obesity and (b) self-reported, multivariate-adjusted dietary carbohydrate intake from 1971-1975 to 1999-2002 (NHANES - National Health and Nutrition Examination Survey) the later surveys. The interaction of PIR with survey $(P<0.001)$ was noted for the percentage reporting a fruit or a DDS of 5, and energy-adjusted amount of vitamin C (Fig. 2). In NHANES I and II, increasing PIR was associated with higher percentage reporting a fruit or all five food groups, and higher intake of vitamin C; however, these PIR-related differences diminished or disappeared in later surveys. The PIR by survey interaction was also significant for serum TC $(P=0.001)$. Serum TC and PIR were positively associated in NHANES II $(P<0.00001)$; in all other surveys, this association was not significant. (Table 4 provides only the main effect for this variable.) For all other variables examined in this study, the interaction terms were not significant $(P>0.01)$.

\section{Main effects of education and PIR}

Tables 3 and 4 present main effects only for those variables where an interaction was not significant $(P>0.01)$. Across all surveys combined, after adjustment for PIR and or its interaction with survey year, increasing education level was associated $(P<0.001)$
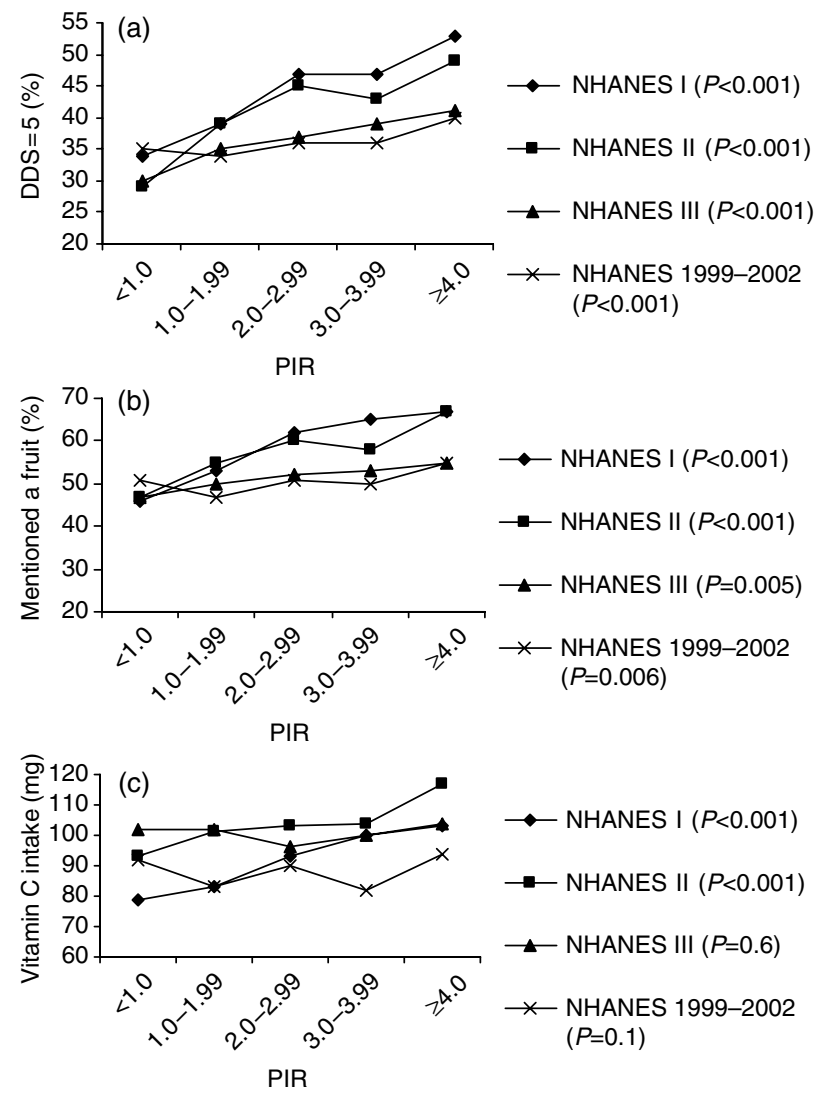

Fig. 2 Changes in the association of poverty income ratio (PIR) with self-reported, multivariate-adjusted dietary attributes from 1971-1975 to 1999-2002: (a) reporting a dietary diversity score (DDS) of 5; (b) reporting a fruit; (c) energy-adjusted vitamin C intake (NHANES - National Health and Nutrition Examination Survey) 
Table 3 Adjusted mean $\pm \mathrm{SE}^{*}$ and prevalence of biomarkers and dietary attributes on the recall day, by categories of level of education in the US population, across all surveys (NHANES I, NHANES II, NHANES III and NHANES 1999-2002)

\begin{tabular}{|c|c|c|c|c|}
\hline & \multicolumn{3}{|c|}{ Years of education } & \multirow[b]{2}{*}{$P($ trend $)$} \\
\hline & $<12$ & 12 & $>12$ & \\
\hline$n$ & 14759 & 11361 & 10480 & \\
\hline Serum TC $\left(\mathrm{mg} \mathrm{dl}^{-1}\right) \dagger$ & $213 \pm 0.7$ & $213 \pm 0.7$ & $210 \pm 0.6$ & 0.001 \\
\hline Serum HDL-C (mg dl $\left.{ }^{-1}\right) \dagger \ddagger$ & $50 \pm 0.4$ & $50 \pm 0.3$ & $50.7 \pm 0.3$ & 0.04 \\
\hline Amount of all foods and beverages $(\mathrm{g})$ & $2458 \pm 22$ & $2525 \pm 19$ & $2432 \pm 18$ & 0.17 \\
\hline Energy (kcal) & $2037 \pm 14$ & $2134 \pm 15$ & $2091 \pm 13$ & 0.02 \\
\hline Energy density $\left(\mathrm{kcal} \mathrm{g}^{-1}\right) \S$ & $1.71 \pm 0.01$ & $1.68 \pm 0.01$ & $1.59 \pm 0.01$ & $<0.001$ \\
\hline Total fat $(\mathrm{g}) \boldsymbol{q}$ & $82 \pm 0.6$ & $83 \pm 0.5$ & $82 \pm 0.5$ & 0.44 \\
\hline Saturated fat $(\mathrm{g}) \emptyset$ & $28 \pm 0.2$ & $28 \pm 0.2$ & $28 \pm 0.2$ & 0.53 \\
\hline Vitamin C (mg) & $85 \pm 2$ & $93 \pm 2$ & $108 \pm 2$ & $<0.001$ \\
\hline Vitamin A (IU) & $5253 \pm 142$ & $5504 \pm 106$ & $6608 \pm 140$ & $<0.001$ \\
\hline Calcium (mg) & $749 \pm 8$ & $776 \pm 9$ & $832 \pm 8$ & $<0.001$ \\
\hline Potassium (mg)ף & $2659 \pm 17$ & $2649 \pm 18$ & $2742 \pm 16$ & $<0.001$ \\
\hline Mentioned any fruit (\%) & $45 \pm 0.8$ & $53 \pm 0.7$ & $63 \pm 0.7$ & $<0.001$ \\
\hline Mentioned any vegetable (\%) & $89 \pm 0.4$ & $92 \pm 0.3$ & $93 \pm 0.3$ & $<0.001$ \\
\hline $\mathrm{DDS}=5(\%) \|$ & $31 \pm 0.7$ & $39 \pm 0.7$ & $47 \pm 0.8$ & $<0.001$ \\
\hline
\end{tabular}

SE - standard error; NHANES - National Health and Nutrition Examination Survey; TC, total cholesterol; HDL-C - highdensity lipoprotein cholesterol; DDS - dietary diversity score.

${ }^{*}$ From regression models that included gender, age, age $^{2}$, race/ethnicity (white, black, other), years of education ( $<12,12$, $>12)$, poverty income ratio $(<1.0,1.0-1.99,2.0-2.99,3.0-3.99, \geq 4.0)$ survey (NHANES I, II, III, 1999-2002) and poverty income ratio by survey interaction (mentioned any fruit, DDS $=5$ and vitamin C) $(n=36600)$.

† Models included smoking status (never, former, current), alcohol user (yes, no) and any leisure-time physical activity (yes, no) in addition to above variables. Included respondents with complete covariate information $(n=33809$ for serum TC; 23685 for serum HDL-C).

† HDL-C analyses were based on data from NHANES II, III and 1999-2002 (HDL-C data were not available for NHANES I).

$\S$ Energy density $=\mathrm{kcal} \mathrm{g}^{-1}$ of all reported foods and nutritive beverages.

I Models included energy intake (kcal).

\| DDS based on consideration of mention of all five food groups (dairy, fruit, vegetable, grain, meat or alternative) in the recall.

with lower serum TC and energy density, but higher intakes of vitamins $\mathrm{A}$ and $\mathrm{C}$, calcium and potassium, and reporting of a fruit, a vegetable or a DDS of 5 (Table 3). Higher PIR was similarly associated
$(P<0.001)$ with lower BMI, carbohydrate intake and energy density, but higher amount of all foods and beverages, intakes of energy and potassium, and reporting of a vegetable in the recall (Table 4).

Table 4 Adjusted mean $\pm \mathrm{SE}^{*}$ and prevalence of biomarkers and dietary attributes on the recall day, by categories of level of PIR in the US population, across all surveys (NHANES I, NHANES II, NHANES III and NHANES 1999-2002)

\begin{tabular}{|c|c|c|c|c|c|c|}
\hline & \multicolumn{6}{|c|}{ PIR } \\
\hline & $<1.0$ & $1.0-1.99$ & $2.0-2.99$ & $3.0-3.99$ & $\geq 4.0$ & $P($ trend) \\
\hline$n$ & 6222 & 9578 & 7586 & 5729 & 7485 & \\
\hline $\mathrm{BMI} \geq 30 \mathrm{~kg} \mathrm{~m}^{-2}(\%) \dagger$ & $26 \pm 1$ & $25 \pm 0.8$ & $24 \pm 0.8$ & $21 \pm 0.8$ & $20 \pm 0.7$ & $<0.001$ \\
\hline Serum TC $\left(\mathrm{mg} \mathrm{dl}^{-1}\right) \dagger$ & $210 \pm 1.1$ & $211 \pm 0.9$ & $213 \pm 0.7$ & $213 \pm 0.9$ & $211 \pm 0.6$ & 0.24 \\
\hline Serum HDL-C $\left(\mathrm{mg} \mathrm{dl}^{-1}\right) \dagger \ddagger$ & $49.2 \pm 0.4$ & $50.2 \pm 0.3$ & $49.9 \pm 0.3$ & $50.2 \pm 0.3$ & $51.1 \pm 0.3$ & 0.002 \\
\hline Amount of all foods and beverages ( $\mathrm{g}$ ) & $2366 \pm 30$ & $2454 \pm 23$ & $2451 \pm 21$ & $2488 \pm 18$ & $2518 \pm 19$ & $<0.001$ \\
\hline Energy (kcal) & $2010 \pm 22$ & $2081 \pm 17$ & $2103 \pm 15$ & $2110 \pm 14$ & $2106 \pm 16$ & $<0.001$ \\
\hline Energy density $\left(\mathrm{kcal} \mathrm{g}^{-1}\right) \S$ & $1.65 \pm 0.02$ & $1.68 \pm 0.01$ & $1.66 \pm 0.01$ & $1.65 \pm 0.01$ & $1.62 \pm 0.01$ & 0.003 \\
\hline Carbohydrate $(\mathrm{g}) \boldsymbol{q}$ & $247 \pm 3$ & $248 \pm 2$ & $242 \pm 1$ & $243 \pm 2$ & $237 \pm 1$ & $<0.001$ \\
\hline Total fat $(\mathrm{g})$ q & $82 \pm 0.7$ & $82 \pm 0.5$ & $84 \pm 0.6$ & $82 \pm 0.7$ & $82 \pm 0.5$ & 0.92 \\
\hline Saturated fat $(\mathrm{g}) \boldsymbol{q}$ & $28 \pm 0.3$ & $28 \pm 0.2$ & $29 \pm 0.2$ & $28 \pm 0.3$ & $28 \pm 0.2$ & 0.18 \\
\hline Vitamin A (IU) & $5522 \pm 193$ & $5667 \pm 175$ & $5839 \pm 155$ & $5850 \pm 163$ & $6212 \pm 135$ & 0.01 \\
\hline Calcium (mg) & $797 \pm 13$ & $793 \pm 11$ & $798 \pm 9$ & $779 \pm 9$ & $790 \pm 9$ & 0.42 \\
\hline Potassium (mg)ף & $2601 \pm 21$ & $2612 \pm 22$ & $2656 \pm 19$ & $2657 \pm 20$ & $2733 \pm 17$ & $<0.001$ \\
\hline Mentioned any vegetable (\%) & $87 \pm 1$ & $88 \pm 0.5$ & $92 \pm 0.5$ & $93 \pm 0.5$ & $93 \pm 0.4$ & $<0.001$ \\
\hline
\end{tabular}

SE - standard error; PIR - poverty income ratio; NHANES - National Health and Nutrition Examination Survey; BMI - body mass index; TC, total cholesterol; HDL-C - high-density lipoprotein cholesterol; DDS - dietary diversity score.

${ }^{*}$ From regression models that included gender, age, age ${ }^{2}$, race/ethnicity (white, black, other), years of education $(<12,12,>12), \mathrm{PIR}(<1,1.0-1.99,2.0-$ $2.99,3.0-3.99, \geq 4.0$ ) survey (NHANES I, II, III, 1999-2002) and education by survey interaction (carbohydrate, BMI).

† Models included BMI (for serum TC and HDL-C), smoking status (never, former, current), alcohol user (yes, no) and any leisure-time physical activity (yes, no) in addition to above variables. Included respondents with complete covariate information ( $n=34993$ for BMl; 33809 for serum TC; 23685 for serum HDL-C).

‡ HDL-C analyses were based on data from NHANES II, III and 1999-2002 (HDL-C data were not available for NHANES I).

$\S$ Energy density $=\mathrm{kcal} \mathrm{g}^{-1}$ of all reported foods and nutritive beverages.

q Models included energy intake (kcal). 


\section{Discussion}

The data presented reveal that SEP differentials in selfreported dietary attributes and biomarkers examined in this study did not increase in the US population from 1971 to 2002. However, SEP differentials in most examined outcomes persisted over three decades. Diminished SEP differentials were seen for only a few of the outcomes and may reflect population-wide shifts in intake (discussed below). Therefore, while our findings do not support increasing SEP differential in diet quality as a contributor to the increasing SEP differential in mortality, they do suggest the continued importance of diet in addressing educationand income-related disparity in health of the US population.

The education differential in the energy-adjusted amount of carbohydrate and the PIR differential in mention of a fruit or all five food groups (DDS of 5) and vitamin C intake, noted in 1971-1980, declined from 1988 to 2002. The narrowing of the education differential in carbohydrate intake appears to reflect a population-wide increase in the intake of this macronutrient rather than a selective change by the low-education group. Similarly, narrowing of the PIR-associated gap in mention of a fruit or all five food groups does not appear to be due to gains by respondents in low PIR categories, but rather because of lower reporting of these food groups by higher PIR respondents in later surveys. Nevertheless, we cannot exclude the possibility of SEP differences in types of carbohydrates or of foods within each of the five food groups, as our methods were not sensitive to these differences. For example, it is possible that, within a food group, food selections (such as fish vs. hamburger, fresh fruits vs. canned fruits, or whole grains vs. refined grains) may differ by SEP categories, and their patterns of consumption may also have changed over time. These possible SEP differences in quality of food choices require further study.

In our study, both measures of SEP were independent predictors of several dietary attributes. However, relative to PIR as operationalised in this study, not only were education differentials present for most of the examined dietary and biomarker outcomes, but also the magnitude of these differentials was larger. Although total energy intake or amount of foods reported appeared to differ little by education, the intake of micronutrients examined in the study (vitamins A and C, potassium and calcium), and mention of fruits, vegetables or overall diet quality (DDS), were lower in Americans with a lower level of education in all surveys. This suggests that in all surveys, food selections reported by respondents with higher education differed from those of respondents with lower education level. Conversely, increasing PIR was accompanied by an increase in total amount of reported foods and energy intake, but not all micronutrients. Education and PIR are known to be correlated. However, the two indicators are able to capture different dimensions of dietary behaviours, which may explain the different associations of education and income with the outcomes examined in this study. While income may be related to diet by affecting purchasing ability for foods recommended in dietary guidance, this gap can be somewhat narrowed by food assistance programmes. The level of education, however, can affect not only purchasing ability, but also may be associated with exposure to and understanding of dietary information, which in turn may relate to motivation for acquisition of behaviours promoted in dietary guidance. To close the education-related gaps in dietary quality, it is important that dietary guidance-related messages be simple and use media that are accessible to Americans with a lower level of education. Moreover, food assistance programmes should include nutrition education components.

Reports from the Nationwide Food Consumption Survey, 1977-1978 and NHANES II, 1976-1980 found little variation in unadjusted dietary nutrient intakes by household income or level of education ${ }^{31,32}$; although other reports have provided evidence of a SEP differential in dietary intakes ${ }^{33-42}$. Relatively few studies, however, have examined time trends in the association of dietary intake with $\mathrm{SEP}^{43-46}$. With one exception ${ }^{46}$, most of the published reports on time trends in SEP and diet association have studied populations in different European countries ${ }^{43-45}$. A comparison of our results with findings from other studies is complicated by differences in methods of dietary assessment, time period covered in the trend analysis, operational forms of dietary variables and measures of SEP, and analytic methodology. Despite these differences, the results of most of these studies ${ }^{43-45}$ are concordant with the results reported here. The lack of an association of dietary total and saturated fat intake with measures of SEP in our study is in accord with other such reports from Europe ${ }^{43,45}$ and the USA ${ }^{46}$. Data from the Dutch National Food Consumption surveys revealed persistence of socio-economic status-related differences in micronutrient intake in the period from 1987 to $1997^{44}$. Conversely, using food consumption data from the UK, James et al. concluded that the socio-economic gap in consumption of fruits, vegetables and vitamin $\mathrm{C}$ widened over the 15-year span from 1980 to $1995^{12}$.

We found the strong PIR differential in the likelihood of being obese to be unchanged from 1971-1975 to 19992002; however, the education-related differential narrowed in 1999-2002. Zhang and Wang also examined trends in the association of education and obesity in 2060-year-olds using data from NHANES I to NHANES 19992000, and found that the education differential in risk of obesity had declined in the last survey ${ }^{47}$. We observed similar trends, although Zhang and Wang did not formally test for the change in the association of education with obesity across surveys, nor did they adjust for the effects of several known correlates of body weight.

James et $a l .^{12}$ and Drewnowski et al. ${ }^{48}$ have argued that in affluent nations, the diet quality of lower-income 
consumers compares unfavourably with that of higherincome consumers because diets rich in protective nutrients cost more. In an analysis of French diets, high sugar and fat intakes were associated with lower overall diet cost but higher energy density ${ }^{41}$. Thus, diets with high energy density may be consumed in association with low SEP and may be implicated in a higher risk of obesity in these consumers. Our results are supportive of the notion that low-SEP consumers have diets of higher energy density. Education and PIR were independent predictors of dietary energy density in all four surveys.

We would like to note the following limitations of our study. First, the methods used to collect the 24-hour dietary recall in NHANES have changed over the course of the four surveys ${ }^{14}$. These include a change in methods used by dietary interviewers to administer the 24-hour recall, and use of multiple-pass and probing to improve the recalls. Second, the database on nutrient composition of foods has expanded and values of many nutrients may have changed because of improved analytical techniques and food sampling methods ${ }^{49}$. Third, the recalls obtained in NHANES I and II were limited to weekdays, whereas weekend days were included in later surveys ${ }^{14}$. Because the NCHS did not conduct any bridging studies to determine the systematic effect of changes in dietary methodology on food and nutrient intakes, the confounding of time effect with the method effect remains a possibility. For these reasons, we urge caution in interpretation of secular trends across surveys presented in Table 2. Within each survey, however, the methods used for data collected from all respondents in all potential exposure categories of income or education were similar. Therefore, while these data may be of limited value in estimating survey effect, they are valid for an examination of changes in the association of measures of SEP with dietary attributes in these surveys. To our knowledge, there are no data to help us examine the possibility that change in methodology may differentially affect those with different PIR or education.

We also note that because Hispanics were included with whites in the NHANES I and II public release data, we grouped them similarly in later surveys. Because the distribution of Hispanics in the US population has changed over the period of these surveys, this may have biased the results of secular trends.

Measurement error has been recognised as a problem in all methods of assessing dietary intake, including 24-hour recalls used in the NHANES ${ }^{50,51}$. Low energy reporting has been noted in NHANES II and NHANES III ${ }^{52-54}$, and is more likely to occur in conjunction with low income and low level of education ${ }^{55}$. In an attempt to explore this issue, we examined trends in the association of PIR and education with the ratio of reported EI to calculated BEE. Using a ratio of $<1.2$ to suggest low energy reporting, the percentage of the population reporting EI/BEE of $<1.2$ decreased over time; but we found no change in the association of PIR or education with the odds of reporting $\mathrm{EI} / \mathrm{BEE}<1.2$ (data not shown). Given the increasing media focus on the association of diet with health, however, it is possible that patterns of reporting of 'nutritionally correct' foods may have changed over time, and the type of reporting errors also may differ by categories of exposure variables. The EI/BEE ratios are of little value in examining these issues, which require further investigation. Due to the lack of repeat measurement of dietary intake in all surveys, we could not compute usual dietary intakes per recent recommendations $^{56}$; therefore, we did not derive estimates of prevalence of nutrient adequacy.

In conclusion, although the income and education differentials in self-reported food and nutrient intake did not increase over time, the persistence of such differences over three decades suggests continued need for improvement in the quality of diets of Americans with a low income and education.

\section{Acknowledgements}

Source of funding: The work was funded in part by the National Institutes of Health, grant award CA108274 (A.K.K.), and the intramural research programme of the Department of Health and Human Services, National Institutes of Health, National Cancer Institute (B.I.G.).

Conflict of interest declaration: None declared.

Authorship responsibilities: A.K.K. was responsible for all aspects of this work: conceptualisation of the study question, study design, operationalisation of exposures and outcomes, data analysis, data interpretation and preparation of the manuscript. B.I.G. provided input on the study design, developed methods for combining data from four national surveys and analytic strategy, and was involved in data interpretation and preparation of the manuscript.

Acknowledgements: We thank Lisa L Kahle for expert assistance with SAS and SUDAAN programming. (Ms Kahle gave permission for this acknowledgement.)

\section{References}

1 US Department of Health and Human Services. Healthy People 2010: Understanding and Improving your Health. Washington, DC: Government Printing Office, 2000.

2 Elo IT, Preston SH. Educational differentials in mortality: United States, 1979-85. Social Science \& Medicine 1996; 42: 47-57.

3 Smith GD, Neaton JD, Wentworth D, Stamler R, Stamler J. Socioeconomic differential in mortality risk among men screened for the Multiple Risk Factor Intervention Trial: I. White men. American Journal of Public Health 1996; 86: 486-96.

4 Smith GD, Wentworth D, Neaton JD, Stamler R, Stamler J. Socioeconomic differential in mortality risk among men screened for the Multiple Risk Factor Intervention Trial: II. Black men. American Journal of Public Health 1996; 86: 497-504. 
5 Singh GK, Miller BA, Hankey BF, Edwards BK. Area Socioeconomic Variations in US Cancer Incidence, Mortality, Stage, Treatment, Survival, 1975-1999. NCI Cancer Surveillance Monograph Series No. 4; NIH Publication No. 03-5417. Bethesda, MD: National Cancer Institute, 2003.

6 Singh GK, Siahpush M. Increasing inequalities in all-cause and cardiovascular mortality among US adults aged 25-64 years by area socio-economic status. International Journal of Epidemiology 2002; 31: 600-13.

7 Crimmins EM, Saito Y. Trends in healthy life expectancy in the United States, 1970-1990: gender, racial, and educational differences. Social Science \& Medicine 2001; 52: 1629-41.

8 Steenland K, Henley J, Thun M. All-cause and cause-specific death rates by educational status for two million people in two American Cancer Society cohorts, 1959-1996. American Journal of Epidemiology 2002; 156: 11-21.

9 Pappas G, Queen S, Hadden W, Fisher G. The increasing disparity in mortality between socioeconomic groups in the United States, 1960 and 1986. New England Journal of Medicine 1993; 329: 103-9.

10 Fuhrer R, Shipley MJ, Chastang JF, Schmaus A, Niedhammer I, Stansfield SA, et al. Socioeconomic position, health, and possible explanations: a tale of two cohorts. American Journal of Public Health 2002; 92: 1290-4.

11 Smith DG, Brunner E. Socio-economic differentials in health: the role of nutrition. Proceedings of the Nutrition Society 1997; 56: 75-90.

12 James WPT, Nelson M, Ralph A, Leather S. Socioeconomic determinants of health: the contribution of nutrition to inequalities in health. British Medical Journal 1997; 314: $1545-52$.

13 Frazao E. High costs of eating patterns in the US. In: Frazao E, ed. America's Eating Habits: Changes and Consequences. Washington, DC: US Department of Agriculture, 1999; 5-31.

14 Centers for Disease Control and Prevention, National Center for Health Statistics. National Health and Nutrition Examination Survey (NHANES): NHANES I, II, III, 1999-2000 and 2001-2002. Available at http://www.cdc.gov/nchs/about/ major/nhanes/current.htm; http://www.cdc.gov/nchs/ about/major/nhanes/nhanesi.htm; http://www.cdc.gov/ nchs/about/major/nhanes/nhanesii.htm; http://www.cdc. gov/nchs/about/major/nhanes/nh3data.htm; http://www. cdc.gov/nchs/about/major/nhanes/NHANES99_00.htm; http://www.cdc.gov/nchs/about/major/nhanes/nhanes0102.htm

15 Krieger N, Williams DR, Moss NE. Measuring social class in US public health research: concepts, methodologies, and guidelines. Annual Review of Public Health 1997; 18: $341-78$.

16 Adler NE, Marmot M, McEwen BS, Stewart J, eds. Socioeconomic Status and Health in Industrial Nations. New York: New York Academy of Sciences, 1999.

17 Briefel RR, Johnson CL. Secular trends in dietary intake in the United States. Annual Review of Nutrition 2004; 24: 401-31.

18 Poppitt SD, Prentice AM. Energy density and its role in the control of food intake: evidence from metabolic and community studies. Appetite 1996; 26: 153-74.

19 Bell EA, Castellanos VH, Pelkman CL, Thorwart ML, Rolls BJ. Energy density of foods affects energy intake in normalweight women. American Journal of Clinical Nutrition 1998; 67: 412-20.

20 Cox DN, Mela DJ. Determination of energy density of freely selected diets: methodological issues and implications. International Journal of Obesity and Related Metabolic Disorders 2000; 24: 49-54.

21 Kant AK, Graubard BI. Energy density of diets reported by American adults: association with food group intake, nutrient intake, and body weight. International Journal of Obesity 2005; 29: 950-6.
22 Rolls BJ, Bell EA, Thorwart ML. Water incorporated into a food but not served with a food decreases energy intake in lean women. American Journal of Clinical Nutrition 1999; 70: $448-55$.

23 Kant AK, Block G, Schatzkin A, Ziegler RG, Nestle M. Dietary diversity in the US population, NHANES II, 1976-80. Journal of the American Dietetic Association 1991; 91: 1526-31.

24 Kant AK, Schatzkin A, Harris TB, Ziegler R, Block G. Dietary diversity and subsequent mortality in the First National Health and Nutrition Examination Survey Epidemiologic Follow-up Study. American Journal of Clinical Nutrition 1993; 57: 434-40.

25 Kant AK, Graubard BI. A comparison of three dietary pattern indexes for predicting biomarkers of diet and disease. Journal of the American College of Nutrition 2005; 24: 294-303.

26 Institute of Medicine. Dietary Reference Intakes for Energy, Carbohydrate, Fiber, Fat, Fatty Acids, Cholesterol, Protein, and Amino Acids. Washington, DC: National Academy Press, 2002/2005

27 Flegal KM, Graubard BI, Williamson DF, Gail MH. Excess deaths associated with underweight, overweight, and obesity. Journal of the American Medical Association 2005; 293: $1861-7$.

28 Korn EL, Graubard BI. Analysis of Health Surveys. New York: John Wiley and Sons, Inc., 1999; 126-9.

29 Korn EL, Graubard BI. Analysis of Health Surveys. New York: John Wiley and Sons, Inc., 1999; 286-92.

30 Research Triangle Institute. SUDAAN User's Manual. Release 8.3. Research Triangle Park, NC: Research Triangle Institute, 2003.

31 Windham CT, Wyse BW, Hansen RG, Hurst RL. Nutrient density of diets in the USDA Nationwide Food Consumption Survey, 1977-1978: 1. Impact of socioeconomic status on dietary density. Journal of the American Dietetic Association 1983; 82: $28-34$.

32 Carroll MS, Abraham S, Dresser CM. Dietary Intake Source Data: United States 1976-1980. Data from the National Health Survey Series 11, No. 231; DHHS Publication No. (PHS) 83-1681. Hyattsville, MD: National Center for Health Statistics, 1983.

33 Kushi LH, Folsom AR, Jacobs DR, Luepker RV, Elmer PJ, Blackburn H. Educational attainment and nutrient consumption patterns: The Minnesota Heart Survey. Journal of the American Dietetic Association 1988; 88: 1230-6.

34 Shimakawa T, Sorlie P, Carpenter MA, Dennis B, Tell GS, Watson R, et al. for ARIC study investigators. Dietary intake patterns and sociodemographic factors in the atherosclerosis risk in communities study. Preventive Medicine 1994; 23: 769-80.

35 Drewnowski A. Fat and sugar: an economic analysis. Journal of Nutrition 2003; 133: 838S-40S.

36 Roos E, Prattala R, Lahelma E, Kleemola P, Pietinen P. Modern and healthy? Socioeconomic differences in the quality of diet. European Journal of Clinical Nutrition 1996; 50: 753-60.

37 Van Rossum CTM, van de Mheen H, Witteman JCM, Grobbee E, Makenbach JE. Education and nutrient intake in Dutch elderly people. The Rotterdam Study. European Journal of Clinical Nutrition 2000; 54: 159-65.

38 Groth MV, Fagt S, Brondsted L. Social determinants of dietary habits in Denmark. European Journal of Clinical Nutrition 2001; 55: 959-66.

39 Irala-Estevez JD, Groth M, Johansson L, Oltersdorf U, Prattala R, Martinez-Gonzalez MA. A systematic review of socio-economic differences in food habits in Europe: consumption of fruits and vegetables. European Journal of Clinical Nutrition 2000; 54: 706-14.

40 Hupkens CLH, Knibe RA, Drop MJ. Social class differences in women's fat and fiber consumption. A cross-national study. Appetite 1997; 28: 131-49. 
41 Darmon N, Ferguson EL, Briend A. A cost constraint alone has adverse effects on food selection and nutrient density: an analysis of human diets by linear programming. Journal of Nutrition 2002; 132: 3764-71.

42 Friel S, Kelleher CC, Nolan G, Harrington J. Social diversity of Irish adults nutritional intake. European Journal of Clinical Nutrition 2003; 57: 865-75.

43 Prattala R, Berg M-A, Puska P. Diminishing or increasing contrasts? Social class variation in Finnish food consumption patterns, 1979-1990. European Journal of Clinical Nutrition 1992; 46: 279-87.

44 Hulshof KFAM, Brussard JH, Kruizinga AG, Telman J, Lowik MRH. Socio-economic status, dietary intake and $10 \mathrm{y}$ trends: the Dutch National Food Consumption Survey. European Journal of Clinical Nutrition 2003; 57: 128-37.

45 Perrin AE, Simon C, Hedelin G, Arveiler D, Schaffer P, Schlienger JL. Ten-year trends of dietary intake in middleaged French population: relationship with educational level. European Journal of Clinical Nutrition 2002; 56: 393-401.

46 Popkin BM, Siega-Riz AM, Haines PS. A comparison of dietary trends among racial and socioeconomic groups in the United States. New England Journal of Medicine 1996; 335: 716-20 [Published correction in New England Journal of Medicine 1997; 337: 1846-8.]

47 Zhang Q, Wang Y. Trends in the association between obesity and socioeconomic status in US adults: 1971-2000. Obesity Research 2004; 12: 1622-32.

48 Drewnowski A, Darmon N, Briend A. Replacing fats and sweets with vegetables and fruits: question of cost. American Journal of Public Health 2004; 94: 1555-9.
49 Anderson E, Perloff B, Ahuja JKC, Raper N. Tracking nutrient changes for trends analysis in the United States. Journal of Food Composition and Analysis 2001; 14: 287-94.

50 Bingham SA. The dietary assessment of individuals, new techniques and recommendations. Nutrition Abstracts and Reviews 1987; 57: 704-42.

51 Livingstone MBE. Assessment of food intakes: are we measuring what people eat? British Journal of Biomedical Science 1995; 52: 58-67.

52 Klesges RC, Eck LH, Ray JW. Who underreports dietary intake in a dietary recall? Evidence from the second National Health and Nutrition Examination Survey. Journal of Consulting and Clinical Psychology 1995; 63: 438-44.

53 Briefel RR, Sempos CT, McDowell MA, Chien S, Alaimo K. Dietary methods research in the third National Health and Nutrition Examination Survey: underreporting of energy intake. American Journal of Clinical Nutrition 1997; 65: 203S-9S.

54 Kant AK. The nature of dietary reporting by adults in the Third National Health and Nutrition Examination Survey, 1988-94. Journal of the American College of Nutrition 2002; 21: $315-27$.

55 Pryer JA, Vrijheid M, Nichols R, Kiggins M, Elliott P. Who are the low energy reporters in the dietary and nutritional survey of British adults? International Journal of Epidemiology 1997; 29: 146-54.

56 Food and Nutrition Board, Institute of Medicine. Dietary Reference Intakes. Applications in Dietary Assessments Washington, DC: National Academy Press, 2001. 